Time and strain-specific downregulation of intestinal EPAS1 via miR-148a by

\title{
Bifidobacterium bifidum
}

Amel Taibi ${ }^{1}$, Natasha Singh ${ }^{1}$, Jianmin Chen ${ }^{1}$, Stefania Arioli ${ }^{1,2}$, Simone Guglielmetti $^{2}$ and Elena M. Comelli ${ }^{1,3 \#}$

${ }^{1}$ Department of Nutritional Sciences and ${ }^{3}$ Centre for Child Nutrition and Health, Faculty of Medicine, University of Toronto, ON, Canada

${ }^{2}$ Department of Food, Environmental and Nutritional Sciences (DeFENS), University of Milan, 20133 Milan, Italy;

\# Corresponding Author; Mailing address: Department of Nutritional Sciences, Faculty of Medicine, University of Toronto, Fitzgerald Building room 308a, 150 College Street, M5S3E2, Toronto, ON, Canada. Phone: +1 416978 6284; E-mail:

elena.comelli@utoronto.ca

Keywords: probiotic, Bifidobacterium bifidum, microRNA, intestine, EPAS1, time

\section{ABSTRACT}

Scope: Bifidobacteria play a role in intestinal homeostasis but molecular mechanisms remain under-investigated. The aim of this study was to assess if probiotic Bifidobacterium strains alter expression of intestinal microRNA and downstream target gene response.

Received: 07-14-2016; Revised: 10-07-2016; Accepted: 11-18-2016

This article has been accepted for publication and undergone full peer review but has not been through the copyediting, typesetting, pagination and proofreading process, which may lead to differences between this version and the Version of Record. Please cite this article as doi: 10.1002/mnfr.201600596. 
Methods and results: The expression of miR-148a and its validated target EPASI (endothelial PAS domain protein 1) was analyzed in Caco-2 cells and mice cecum in response to Bifidobacterium bifidum MIMBb75, B. bifidum NCC390 or B. longum NCC2705. In vitro, exposure to B. bifidum MIMBb75, but not to B. bifidum NCC390 or B. longum NCC2705, increased the expression of miR-148a after 1 and 4 hours $(\mathrm{P}<0.01)$, but not after 24h. In vivo, B. bifidum MIMBb75 administration to C57BL/6J mice increased miR-148a expression in the cecum after 2 but not 14 days $(\mathrm{P}<0.05)$. The increase of miR-148a was accompanied by a decrease of EPAS1 expression in Caco-2 cells and cecum $(\mathrm{P}<0.05)$. Silencing of miR-148a reversed B. bifidum MIMBb75-dependent downregulation of EPAS1.

Conclusion: This study shows an early response of intestinal cells to B. bifidum MIMBb75 through miR-148a modulation. This brings a new concept of strain- and time-dependent bifidobacteria-host crosstalk via microRNA. Probiotic B. bifidum MIMBb75 may help attenuating EPAS1 overexpression associated with intestinal inflammation.

\section{Introduction}

Bifidobacteria are autochthonous inhabitants of human and animal intestinal tracts, representing 80 and $25 \%$ of the infant and adult microbiota, respectively (Reviewed in [1]). This genus comprises widely used probiotics whose benefits are either considered common probiotic core benefits, such as their ability to improve gut microbiota composition $[2,3]$ and inhibit pathogen adhesion [4], or are strain specific, particularly, their capability to modulate the host-immune system [5]. This is in line with the ability of bifidobacteria to elicit host responses in a species- and strain- dependent manner [5-7]. Bifidobacterium bifidum MIMBb75 is a probiotic strain isolated from healthy human feces [8], that resides in cecal-

This article is protected by copyright. All rights reserved. 
colonic regions and modulates the composition of the gut microbiota in mice [9]; in a doubleblind placebo-controlled study, it was shown to significantly reduce irritable bowel syndrome (IBS) symptoms and improve general quality of life [10]. B. bifidum MIMBb75 has stronger ability to adhere to intestinal cells in vitro compared to other probiotics such as B. longum NCC2705 and Lactobacillus rhamnosus GG [11]. B. bifidum MIMBb75 possesses several surface proteins that could enable interaction with host cells. These include the bifidobacterial outer protein (BopA), likely involved in the adhesion process [8], and the murine lytic enzyme TgaA, expressed on the outer surface of $B$. bifidum MIMBb75 cells and found to activate dendritic cells and trigger IL-2 production in vitro $[12,13]$. However, the response of the host to $B$. bifidum MIMBb75 remains largely uncharacterized, particularly at the molecular level. Gene expression regulation is a main mechanism underpinning the continuous interaction between intestinal tissues and microbiota. The coexistence of prokaryotic and eukaryotic cells in the intestine results in a microbiota-dependent gene expression program in host cells $[14,15]$. Not surprisingly, this is affected by probiotic administration, including bifidobacteria [16]. We and others have shown that the intestinal microbiota-dependent gene regulation may occur at the post-transcriptional level, via microRNA, in colon and cecum [17, 18]. MicroRNAs (miRNAs) are 20-22 nucleotides long, non-coding RNAs regulating more than $60 \%$ of the human transcriptome [19]. MiRNAs have emerged as fine-tuners of host gene expression contributing to intestinal homeostasis (Reviewed in [20]. Like mRNA, miRNAs are expressed in a time-dependent manner [21, 22], acting as a response timer to a variety of stimuli. The time-specific response can happen early (within a few hours) such as in gastric epithelial cells exposed to Helicobacter pylori (6 hours) [23] or can be delayed (within days), such as in T lymphocytes where miR146a levels were increased after 8 days to stimulation with PMA (phorbol myristate acetate) and

This article is protected by copyright. All rights reserved. 
ionomycin, reaching their highest level of expression after 20 days [24]. MiRNA responses to commensal or probiotic bacteria have not been largely investigated. We found that the presence of the microbiota impacts the intestinal miRNA signature including expression of miR-148a [18]. MiR-148a is a member of the $m i R-148 / 152$ family (reviewed in [25]) known to play an important role in the regulation of the immune response [26]. Here, we studied the impact of probiotic $B$. bifidum MIMBb75 on intestinal expression of miR-148a and the downstream target gene response in vitro and in vivo.

\section{Materials and methods}

\section{Bifidobacteria}

B. bifidum MIMBb75, B. bifidum NCC390 and B. longum NCC2705 were grown overnight at $37^{\circ} \mathrm{C}$ in MRS broth (Difco, Detroit, MI, USA) supplemented with $0.05 \%$ L-cysteine hydrochloride (cMRS) in an anaerobic chamber (Coy Laboratory Products; Grass Lake, MI, USA) containing an atmosphere of $90 \% \mathrm{~N}_{2}, 5 \% \mathrm{H}_{2}$ and $5 \% \mathrm{CO}_{2}$. Prior to use, cells were harvested at $3000 \times g$ for $10 \mathrm{~min}$, washed twice with phosphate-buffered saline (PBS) and quantified microscopically using Neubauer Bright-Line Hemacytometer (Hausser Scientific, Horsham, PA) and by plating on cMRS Agar.

Caco-2 cell culture, co-incubation and transfection experiments

Caco-2 cells were obtained from the American Type Culture Collection (ATCC, Rockville, MD, USA) and were routinely cultured at $37^{\circ} \mathrm{C}$ in Dulbecco's modified Eagle's medium (DMEM), as previously described [8]. Prior to co-incubation with Bifidobacterium strains, the cells were seeded on 6-well culture plates and grown for two weeks post-confluency [8]. The fully differentiated Caco-2 monolayers were washed with PBS buffer and incubated in parallel with each of the test strain re-suspended in DMEM vehicle without antibiotics, supplemented with $100 \mathrm{mM}$ HEPES (GIBCO, Life Technologies, Carlsbad, CA, USA) at

This article is protected by copyright. All rights reserved. 
bacteria:cell ratio of $10: 1$ for 1,4 and $24 \mathrm{~h}$. Vehicle alone was used as a control. After incubation, cells were washed with PBS, scraped off the bottom of the wells, snap-frozen in liquid nitrogen and stored at $-80^{\circ} \mathrm{C}$ until RNA extraction. Cell viability was determined by Trypan Blue exclusion test (GIBCO, Life Technologies, Carlsbad, USA), whereas bacterial viability was evaluated by plating on cMRS Agar both at the time of addition and after incubation with the cells. Experiments were conducted in triplicates.

For transfection with miRNA inhibitors, Caco-2 cells were seeded on 6-well culture plates at a density of $10^{6}$ cell/well to reach 60 to $80 \%$ of confluency at the time of transfection. Cells were transfected with $10 \mathrm{nM}$ of mirVana miR-148a inhibitor (Assay ID: MH10263), mirVana let-7c miRNA inhibitor (positive control, Assay ID: 4464080) (Invitrogen, Life Technologies, Carlsbad, USA), or corresponding vehicle alone (Mock) using lipofectamine RNAiMAX transfection agent and Opti-MEMI reduced serum medium (Invitrogen, life Technologies, Carlsbad, CA, USA), according to the manufacturer's instructions. The transfection efficiency was evaluated with Block-iT Fluorescent Oligo (Invitrogen, Life Technologies, Carlsbad, USA) according to the manufacturer's protocol. At $72 \mathrm{~h}$ posttransfection, miR-148a inhibitor- or mock- transfected cells were incubated with either $B$. bifidum MIMBb75, prepared as described above, at bacteria:cell ratio of 10:1 or vehicle alone, for 1,4 and $24 \mathrm{~h}$.

In vivo probiotic studies

Two studies were conducted. The first, which has been partially described in [9], used twenty eight 5-6 weeks old C57BL/6J male mice purchased from Jackson's Laboratory (Sacramento, CA, USA) and housed at the Division of Comparative Medicine, University of Toronto. After one week acclimatization, mice were randomized into two groups ( $\mathrm{n}=14$ /group) to receive $0.2 \mathrm{ml}$ of PBS containing $10^{8}$ colony forming units (CFU) of $B$.

This article is protected by copyright. All rights reserved. 
bifidum MIMBb75 cells or PBS only daily for 2 or 14 days, via intragastric gavage. The second study used twelve C57BL/6J male mice at the same age and from the same origin as above. Mice received either $B$. bifidum MIMBb75 or PBS for 2 days (n=4-8/group). Viability and quantity of $B$. bifidum MIMBb75 used for gavage were confirmed by plating on cMRS Agar. Cecal tissues and contents were collected immediately after dissection on ice and stored at $-80^{\circ} \mathrm{C}$. All animal experiments were approved by the animal ethics committee at the University of Toronto (Protocol number: 20010228). Microbiota composition analysis was by qPCR using TaqMan assays (Supporting Information Table S1), as we previously described [9].

\section{RNA extraction and real-time quantitative PCR}

Total RNA was isolated from Caco-2 cells and mouse cecum tissues using the mirVana ${ }^{\mathrm{TM}}$ miRNA isolation kit (Ambion, Life Technologies, Carlsbad, USA), as per the manufacturer's instructions. miRNA and mRNA expression analysis was performed as we described previously [18] (miR-148a, Assay ID: 000470; EPAS1, Assay IDs: Hs01026149_m1 and Mm01236112_m1 for Caco-2 cells and mouse cecum, respectively). MiRNA gene expression data were normalized to the endogenous controls RNU6B (Assay ID: 001093) and sno-135 (Assay ID: 001230) for Caco-2 cells and mouse cecum, respectively. Gene expression was normalized to ACTB (Assay IDs: Hs01060665_g1 and Mm02619580_g1 for Caco-2 cells and mouse cecum, respectively). Fold changes were calculated using the $2^{-\Delta \Delta} \mathrm{Ct}$ method [27].

\section{Identification of microRNA targets}

Putative and experimentally validated targets of miR-148a were identified in silico using the miRWalk database which integrates seven different algorithms [28].

Statistical analysis

This article is protected by copyright. All rights reserved. 
Statistical significance of miRNA and mRNA expression in Caco-2 cells was determined by one-way analysis of variance (ANOVA) followed by Tukey's multiple comparison test. Significance of differential miRNA and gene expression mouse cecum was assessed with the Mann-Whitney test. GraphPad Prism software V5.0 (La Jolla, CA, USA) was used for all statistical analyses and values of $\mathrm{p}<0.05$ were considered significant. Data are presented as mean $\pm \mathrm{SD}$.

\section{Results}

Bifidobacteria modify miR-148a expression in a strain-and time-dependent manner in vitro

To investigate the miRNA response to bifidobacteria in intestinal cells, Caco- 2 cells were incubated for 1, 4 or 24 hours with B. bifidum MIMBb75 or B. bifidum NCC390 or B. longum NCC2705. Caco-2 cells viability was $\geq 93 \%$ and viable counts of the three strains were in the range of 2 to $9 \times 10^{7} \mathrm{CFU} /$ well at all time points (data not shown). B. bifidum MIMBb75 elicited an increase in miR-148a expression after 1 and 4 hours $(3.5 \pm 1.3$ and $2.7 \pm 0.7$ fold changes, respectively, compared to the control, $\mathrm{P}<0.01)$ but not after 24 hours. This increase was more pronounced at the earliest time point. MiR-148a did not change in response to $B$. bifidum NCC390 and to B. longum NCC2705 (Figure 1).

\section{B. bifidum MIMBb75 modifies intestinal miR-148a expression in vivo}

To determine whether B. bifidum MIMBb75 modulates host intestinal miRNA expression in vivo, we first used tissue samples from a study we had previously conducted, where mice were daily gavaged with $10^{8} \mathrm{CFU}$ of B. bifidum MIMBb75 or PBS (as a control) for 2 or 14 days [9]. In the cecum of mice receiving probiotic, B. bifidum counts corresponded to $5.4 \pm$ $0.3 \log \mathrm{CFU} / \mathrm{g}$ of contents at day 2, while $C$. coccoides levels were significantly decreased compared to the PBS gavaged mice $(8.2 \pm 0.1$ versus $7.2 \pm 0.7 \log \mathrm{CFU} / \mathrm{g}$ of contents,

This article is protected by copyright. All rights reserved. 
$\mathrm{P}=0.01$ ) (Supporting Information Figure $\mathrm{S} 1$ ); other taxa known to be represented in the gut microbiota, including C. leptum group, were not affected (data not shown). Bacterial counts at day 14 were previously published and showed the same pattern with significantly increased B. bifidum counts and decreased $C$. coccoides levels in the cecum of mice receiving probiotic [9]. B. bifidum counts increased after 14 days of continuous administration; there was no within group difference between $C$. coccoides counts at the two time points. The caecal expression of miR-148a increased after two days of probiotic treatment $(1.4 \pm 0.1$ fold change, $\mathrm{P}=0.002)$ but not after 14 days $(0.9 \pm 0.3$ fold change) (Figure $2 \mathrm{~A})$. Data at day 2 were confirmed in a second independent study showing B. bifidum counts of $6.0 \pm 0.4 \log$ $\mathrm{CFU} / \mathrm{g}$ of cecum content and a significant decrease of $C$. coccoides counts in mice receiving probiotics compared to the control $(6.0 \pm 0.4$ versus $4.4 \pm 0.2 \log \mathrm{CFU} / \mathrm{g}$ of contents, $\mathrm{P}=0.001$ ) (Supporting Information Figure S1). MiR-148a expression was significantly increased $(1.7 \pm 0.2$ fold change, $\mathrm{P}=0.005)$ (Figure $2 \mathrm{~B})$. These data follow a similar pattern as in Caco-2 cells where the expression of host miR-148a is altered in response to B. bifidum MIMBb75 administration with an early response that differs from that at later time-points.

\section{Identification of miR-148a targets}

To address the role of miR-148a in host intestinal response to probiotic B. bifidum MIMBb75, its gene targets were identified using seven different algorithms. Over 1760 predicted putative targets for miR-148a were found by at least five of these (Supporting Information Table S2); 78 experimentally validated targets were identified including EPAS1 (Supporting Information Table S3). This gene encoding for endothelial PAS domain protein 1 (also known as Hypoxia-inducible factor-2 alpha, HIF-2 $\alpha$ ), was identified by five of the seven interrogated algorithms. Previous studies using mesenchymal stem cells had

This article is protected by copyright. All rights reserved. 
experimentally verified that miR-148a controls the expression of EPAS1 [29]. Thus, this gene was selected for additional investigation in vitro and in vivo.

MiR-148a impacts expression of EPAS1 in intestinal epithelial cells exposed to B. bifidum MIMBb75a

To determine if miR-148a regulates the expression of its target gene EPAS1 in Caco-2 cells in response to B. bifidum MIMBb75, miR-148a was inhibited for 3 days. Then, miR-148ainhibited or mock transfected Caco-2 cells were incubated with B. bifidum MIMBb75 for 1, 4 and 24 hours and miR-148a and EPASl expression levels were measured by RT-qPCR. To ensure the miRNA inhibition was successful, miR-148a expression levels were also determined in intestinal cells transfected with miR-148a-inhibitor and incubated in the presence of vehicle alone (DMEM without antibiotics, supplemented with $100 \mathrm{mM}$ HEPES) for 1, 4 and 24 hours. Silencing of miR-148a was efficient since cells transfected with miR148a inhibitor had a $50 \%$ decrease in miR-148a expression compared to control (miR-148a inhibitor) (Figure 3). In cells transfected with miR-148a inhibitor and co-incubated with $B$. bifidum MIMBb75 (miR-148a inh+BB75), silencing was overcome at 4 and 24 hours, showing the ability of $B$. bifidum MIMBb75 to increase the expression of miR-148a. In line with experiments in Figure 1, miR-148a increased after 1 and 4 hours of co-incubation of mock-transfected cells with B. bifidum MIMBb75 (BB75 only).

As shown in Figure 4A, inhibition of miR-148a significantly affected EPAS1 expression compared to the control (miR-148a inhibitor), with more than $200 \%$ increase in gene expression in the miR-148a silenced group in response to the probiotic strain at 4 hours (miR148a inh+BB75) $(\mathrm{P}<0.001)$. This gene was also found to be significantly downregulated in the cecum of mice gavaged with $B$. bifidum MIMBb75 for 2 days $(0.8 \pm 0.1$ fold change,

This article is protected by copyright. All rights reserved. 
$\mathrm{P}=0.004)$ (Figure 4B). This indicates that down-regulation of EPAS1 gene expression by $B$. bifidum MIMBb75 is mediated by miR-148a.

\section{Discussion}

In this study, we showed that the expression of miR-148a is altered in intestinal cells in response to bifidobacteria in a strain and time-dependent manner. In particular, exposure to $B$. bifidum MIMBb75 induces an early upregulation of miR-148a both in vitro and in vivo, which disappears at later time points. Very few studies have investigated the effect of selected probiotic on miRNA expression. L. rhamnosus GG was found to increase the expression of miR-155 and decrease miR-146a in human dendritic cells [30]. Escherichia coli Nissle 1907 increased tight junction protein expression in T84 cells via miR-595, miR483 and miR-203 downregulation [31]. Heat-treated L. paracasei NCC 2461 downregulated miR-27a in peripheral blood mononuclear cells [32]. Enterococcus faecium NCIMB 10415 upregulated miR-423-5p and downregulated its target gene IGLC (immunoglobulin lambda light $\mathrm{C}$ region) in the intestine of piglets [33]. These studies show that different probiotics elicit different miRNA responses. Though, none of them examined the effects of different probiotics on the same miRNA species. We found that, opposite to B. bifidum MIMBb75, $B$. longum NCC2705 and B. bifidum NCC390 did not affect miR-148a expression. The molecular mechanisms behind this strain- specific response are not clear. Recently, $B$. bifidum MIMBb75 whole genome sequencing revealed the presence of a gene named tgaA coding for a murein lytic enzyme TgaA [12]. This peptidoglycan-degrading enzyme was shown to exert an immunomodulatory effect by inducing DCs activation and IL-2 production through its C-terminal CHAP (cysteine histidine-dependent amidohydrolase/peptidase) domain in a strain-dependent manner, with B. bifidum MIMBb75 showing the strongest effect [13]. Therefore, it is tempting to speculate that miRNA-mediated B. bifidum MIMBb75-host

This article is protected by copyright. All rights reserved. 
interaction depends on the CHAP domain of TgaA. Two extracellular proteins encoded by $B$. bifidum, the sortase-dependent pili and the type IV tight adherence locus have been shown to mediate its interaction with the host [34-36]. However, opposite to TgaA, these proteins are encoded by genes identified in B. bifidum core genome [37], and are thus unlikely to explain strain specific responses. Microbial metabolites such as short chain fatty acids may regulate host gene expression epigenetically [38] offering a possible alternate mechanism for host miRNA modulation. The microbiota is a modifier of the intestinal miRNA response to pathogen exposure, in that the expression of miR-148a was reduced in the intestine of conventional, but not germ-free mice, infected with Listeria monocytogenes [39]. B. bifidum MIMBb75 modifies the microbiota, resulting in increased bifidobacteria and decreased $C$. coccoides counts (this study and [9]). Though, this is already evident at day 2, with no differences between day 2 and 14. Thus, there could be a "direct" effect of B. bifidum MIMBb75 which is not mediated by the microbiota. Though, given its complexity and because the effect of $B$. bifidum MIMBb75 administration on its composition and activity have not been completely elucidated yet, future studies including microbiota profiling are necessary.

MiRNAs respond to bacterial stimuli in a time-dependent manner. For example, pri-miR-147 increased in murine peritoneal macrophages after 2, but not 6 hours of exposure to lipopolysaccharide [40] and the expression of miR-146a in gastric epithelial cells increased between 6-9 hours after H. pylori infection, but decreased after $24 \mathrm{~h}$ [23]. However, the deferred response to bacterial exposure has not been investigated in the literature. Early responses have been deemed essential in innate immune responses where a short reaction time is critical for resistance for example to a pathogenic microorganism. On the other hand, delayed responses may play a role in adaptive immunity [24]. Thus, our data suggest that the

This article is protected by copyright. All rights reserved. 
intestine may adapt to $B$. bifidum MIMBb75 following continued exposure. The time of exposure to a probiotic is a critical factor in clinical practice because it dictates the administration protocol. A time-dependent response following probiotic exposure was found in vitro and in vivo (mouse cecum) for B. bifidum PRL2010 impacting the immune response via alteration of the gene expression signature of HT-29 cells with more consistent changes after 4, rather than 1 or 2, hours of co-incubation[41], and in vivo for Lactobacillus reuteri 100-23 impacting inflammatory pathways after 6 but not 21 days of colonization [42]. Taken together with our findings, these data suggest that there may be an immediate intestinal response to probiotic exposure which may vary from that resulting from repetitive reinoculation. This concept aligns with a study showing that, opposite to continuous supplementation, pulse administration of probiotics maintains high expression levels of mucin 3 in the jejunum and ileum of rats [43].

Based on in silico analysis, several genes were identified as potential targets of miR-148a, including experimentally validated $E P A S 1$. This is a hypoxia-inducible transcription factor of the basic helix-loop-helix/PAS domain family, also known as Hypoxia Inducible Factor-2 $\alpha$ (HIF-2 $\alpha$ ) [44]. High levels of EPAS1 have been associated with digestive system cancers (Reviewed in [45]), IBD [46] and colitis [47]. In a mouse model, EPAS1 was found to activate mediators of inflammation such as TNF- $\alpha$ and to promote colonic inflammation and spontaneous colitis after Citrobacter rodentium infection or dextran sulfate sodium (DSS) administration [47]. Moreover, it has a role in activating iron transporters [48] and intestinal knockout of EPAS1 significantly decreased tissue iron accumulation, due to a decrease in iron absorptive genes, in a mouse model of sickle cell disease (SDC) [49]. Therefore, intestinal EPAS1 has been suggested as a therapeutic target for IBD [47], SCD [49] and different types of cancers [50]. Here, we found that B. bifidum MIMBb75 increased miR-

This article is protected by copyright. All rights reserved. 
148a resulting in down-regulation of EPAS1 in colonic cells. This suggests that B. bifidum MIMBb75 could be considered for maintenance of, or to restore, intestinal homeostasis.

In conclusion, our study brings a new concept of strain specific bifidobacteria-host crosstalk mediated by microRNA. Our findings show that B. bifidum MIMBb75 stimulates gene expression changes in the host at both the transcriptional and miRNA levels in a timedependent manner. Although speculative at this stage, the use of B. bifidum MIMBb75 may be considered to inhibit EPAS1 function in intestinal cells and as a consequence help in preventing or mitigating colitis and other diseases. Molecular antagonists of EPAS1 have been identified [51], providing therapeutic opportunities to its inactivation in disease. Our study is the first to show an alternative way to achieve EPAS1 inhibition in intestinal cells that is mediated by miRNA in response to a probiotic. This warrants further research to better elucidate the mechanisms involved. In addition, timing of probiotic administration may play an important role in clinical practice.

\section{Author contributions}

EMC conceived the study; AT, NS, SA, SG and EMC designed the experiments; AT, NS, JC and SA conducted the experiments; AT and EMC wrote the manuscript; all authors contributed to, read and approved the manuscript; EMC had primary responsibility for the study.

\section{Acknowledgments}

The authors would like to thank Christopher Villa for technical help. This study was supported by the Natural Sciences and Engineering Research Council of Canada (NSERC) (Grant \# RGPIN 356124-2013), the J. P. Bickell Foundation and the James H. Cummings

This article is protected by copyright. All rights reserved. 
Foundation. Elena M. Comelli holds the Lawson Family Chair in Microbiome Nutrition Research at the University of Toronto. Natasha Singh was the recipient of a NSERC Alexander Graham Bell Canada Graduate Scholarship.

Conflict of interest: The authors have declared no conflict of interest

\section{References}

[1] Picard, C., Fioramonti, J., Francois, A., Robinson, T., et al., Review article: bifidobacteria as probiotic agents -- physiological effects and clinical benefits. Alimentary pharmacology \& therapeutics 2005, 22, 495-512.

[2] Bartosch, S., Woodmansey, E. J., Paterson, J. C., McMurdo, M. E., Macfarlane, G. T., Microbiological effects of consuming a synbiotic containing Bifidobacterium bifidum, Bifidobacterium lactis, and oligofructose in elderly persons, determined by real-time polymerase chain reaction and counting of viable bacteria. Clin Infect Dis 2005, 40, 28-37.

[3] Ishizeki, S., Sugita, M., Takata, M., Yaeshima, T., Effect of administration of bifidobacteria on intestinal microbiota in low-birth-weight infants and transition of administered bifidobacteria: a comparison between one-species and three-species administration. Anaerobe 2013, 23, 38-44.

[4] Gagnon, M., Kheadr, E. E., Le Blay, G., Fliss, I., In vitro inhibition of Escherichia coli O157:H7 by bifidobacterial strains of human origin. International journal of food microbiology 2004, 92, 69-78.

[5] Fu, Y. R., Yi, Z. J., Pei, J. L., Guan, S., Effects of Bifidobacterium bifidum on adaptive immune senescence in aging mice. Microbiol Immunol 2010, 54, 578-583.

This article is protected by copyright. All rights reserved. 
[6] Boesten, R., Schuren, F., Willemsen, L., Vriesema, A., et al., Bifidobacterium breve HT-29 cell line interaction: modulation of TNF- $\alpha$ induced gene expression. Beneficial Microbes 2011, 2, 115-128.

[7] Konieczna, P., Akdis, C. A., Quigley, E. M., Shanahan, F., O'Mahony, L., Portrait of an immunoregulatory Bifidobacterium. Gut Microbes 2012, 3, 261-266.

[8] Guglielmetti, S., Tamagnini, I., Mora, D., Minuzzo, M., et al., Implication of an outer surface lipoprotein in adhesion of Bifidobacterium bifidum to Caco-2 cells. Applied and environmental microbiology 2008, 74, 4695-4702.

[9] Singh, N., Arioli, S., Wang, A., Villa, C. R., et al., Impact of Bifidobacterium bifidum MIMBb75 on mouse intestinal microorganisms. FEMS microbiology ecology 2013, 85, 369375.

[10] Guglielmetti, S., Mora, D., Gschwender, M., Popp, K., Randomised clinical trial: Bifidobacterium bifidum MIMBb75 significantly alleviates irritable bowel syndrome and improves quality of life--a double-blind, placebo-controlled study. Alimentary pharmacology \& therapeutics $2011,33,1123-1132$

[11] Guglielmetti, S., Tamagnini, I., Minuzzo, M., Arioli, S., et al., Study of the Adhesion of Bifidobacterium bifidum MIMBb75 to Human Intestinal Cell Lines. Current Microbiology 2009, 59, 167-172.

[12] Guglielmetti, S., Balzaretti, S., Taverniti, V., Miriani, M., et al., TgaA, a VirB1-like component belonging to a putative type IV secretion system of Bifidobacterium bifidum MIMBb75. Applied and environmental microbiology 2014, 80, 5161-5169.

[13] Guglielmetti, S., Zanoni, I., Balzaretti, S., Miriani, M., et al., Murein lytic enzyme TgaA of Bifidobacterium bifidum MIMBb75 modulates dendritic cell maturation through its

This article is protected by copyright. All rights reserved. 
cysteine- and histidine-dependent amidohydrolase/peptidase (CHAP) amidase domain. Applied and environmental microbiology 2014, 80, 5170-5177.

[14] Chen, L., Zhang, Y.-H., Huang, T., Cai, Y.-D., Gene expression profiling gut microbiota in different races of humans. Scientific Reports 2016, 6, 23075.

[15] Reikvam, D. H., Erofeev, A., Sandvik, A., Grcic, V., et al., Depletion of murine intestinal microbiota: Effects on gut mucosa and epithelial gene expression. PloS one 2011, 6, e17996.

[16] Menard, O., Butel, M. J., Gaboriau-Routhiau, V., Waligora-Dupriet, A. J., Gnotobiotic mouse immune response induced by Bifidobacterium sp. strains isolated from infants. Applied and environmental microbiology 2008, 74, 660-666.

[17] Dalmasso, G., Nguyen, H. T., Yan, Y., Laroui, H., et al., Microbiota modulate host gene expression via microRNAs. PloS one 2011, 6, e19293.

[18] Singh, N., Shirdel, E. A., Waldron, L., Zhang, R. H., et al., The murine caecal microRNA signature depends on the presence of the endogenous microbiota. Int J Biol Sci 2012, 8, 171-186.

[19] Friedman, R. C., Farh, K. K., Burge, C. B., Bartel, D. P., Most mammalian mRNAs are conserved targets of microRNAs. Genome research 2009, 19, 92-105.

[20] Runtsch, M. C., Round, J. L., O'Connell, R. M., MicroRNAs and the regulation of intestinal homeostasis. Front Genet 2013, 5, 347.

[21] Moschos, S. A., Williams Ae Fau - Perry, M. M., Perry Mm Fau - Birrell, M. A., Birrell Ma Fau - Belvisi, M. G., et al., Expression profiling in vivo demonstrates rapid changes in lung microRNA levels following lipopolysaccharide-induced inflammation but not in the anti-inflammatory action of glucocorticoids. 2007.

This article is protected by copyright. All rights reserved. 
[22] Koufaris, C., Wright, J., Osborne, M., Currie, R. A., Gooderham, N. J., Time and dosedependent effects of phenobarbital on the rat liver miRNAome. Toxicology 2013, 314, 247253.

[23] Liu, Z., Xiao, B., Tang, B., Li, B., et al., Up-regulated microRNA-146a negatively modulate Helicobacter pylori-induced inflammatory response in human gastric epithelial cells. Microbes and Infection 2010, 12, 854-863.

[24] Curtale, G., Citarella, F., Carissimi, C., Goldoni, M., et al., An emerging player in the adaptive immune response: microRNA-146a is a modulator of IL-2 expression and activation-induced cell death in T lymphocytes. Blood 2010, 115, 265-273.

[25] Chen, Y., Song, Y. X., Wang, Z. N., The microRNA-148/152 family: multi-faceted players. Mol Cancer 2013, 12, 43.

[26] Liu, X., Zhan, Z., Xu, L., Ma, F., et al., MicroRNA-148/152 impair innate response and antigen presentation of TLR-triggered dendritic cells by targeting CaMKIIalpha. J Immunol $2010,185,7244-7251$.

[27] Livak, K. J., Schmittgen, T. D., Analysis of relative gene expression data using real-time quantitative PCR and the 2(-Delta Delta C(T)) Method. Methods (San Diego, Calif 2001, 25, 402-408.

[28] Dweep, H., Sticht, C., Pandey, P., Gretz, N., miRWalk - Database: Prediction of possible miRNA binding sites by "walking" the genes of three genomes. Journal of biomedical informatics 2011.

[29] Giraud-Triboult, K., Rochon-Beaucourt, C., Nissan, X., Champon, B., et al., Combined mRNA and microRNA profiling reveals that miR-148a and miR-20b control human mesenchymal stem cell phenotype via EPAS1. Physiol Genomics 2011, 43, 77-86.

This article is protected by copyright. All rights reserved. 
[30] Giahi, L., Aumueller, E., Elmadfa, I., Haslberger, A. G., Regulation of TLR4, p38 MAPkinase, IkappaB and miRNAs by inactivated strains of lactobacilli in human dendritic cells. Beneficial Microbes 2012, 3, 91-98.

[31] Veltman, K., Hummel, S., Cichon, C., Sonnenborn, U., Schmidt, M. A., Identification of specific miRNAs targeting proteins of the apical junctional complex that simulate the probiotic effect of E. coli Nissle 1917 on T84 epithelial cells. Int J Biochem Cell Biol 2012, 44, 341-349.

[32] Demont, A., Hacini-Rachinel, F., Doucet-Ladeveze, R., Ngom-Bru, C., et al., Live and heat-treated probiotics differently modulate IL10 mRNA stabilization and microRNA expression. J Allergy Clin Immunol 2015, 137, 1264-1267 e1210.

[33] Kreuzer-Redmer, S., Bekurtz, J. C., Arends, D., Bortfeldt, R., et al., Feeding of Enterococcus faecium NCIMB 10415 leads to intestinal miRNA-423-5p-induced regulation of immune-relevant genes. Applied and environmental microbiology 2016, 82, 2263-2269.

[34] O'Connell Motherway, M., Zomer, A., Leahy, S. C., Reunanen, J., et al., Functional genome analysis of Bifidobacterium breve UCC2003 reveals type IVb tight adherence (Tad) pili as an essential and conserved host-colonization factor. Proceedings of the National Academy of Sciences of the United States of America 2011, 108, 11217-11222.

[35] Turroni, F., Serafini, F., Foroni, E., Duranti, S., et al., Role of sortase-dependent pili of Bifidobacterium bifidum PRL2010 in modulating bacterium-host interactions. Proceedings of the National Academy of Sciences of the United States of America 2013, 110, 11151-11156.

[36] Turroni, F., Serafini, F., Mangifesta, M., Arioli, S., et al., Expression of sortasedependent pili of Bifidobacterium bifidum PRL2010 in response to environmental gut conditions. FEMS Microbiol Lett 2014, 357, 23-33.

This article is protected by copyright. All rights reserved. 
[37] Duranti, S., Milani, C., Lugli, G. A., Turroni, F., et al., Insights from genomes of representatives of the human gut commensal Bifidobacterium bifidum. Environ Microbiol $2015,17,2515-2531$.

[38] Kumar, M., Nagpal, R., Verma, V., Kumar, A., et al., Probiotic metabolites as epigenetic targets in the prevention of colon cancer. Nutrition reviews 2013, 71, 23-34.

[39] Archambaud, C., Sismeiro, O., Toedling, J., Soubigou, G., et al., The intestinal microbiota interferes with the microRNA response upon oral Listeria infection. MBio 2013, 4, e00707-00713.

[40] Liu, G., Friggeri, A., Yang, Y., Park, Y. J., et al., miR-147, a microRNA that is induced upon Toll-like receptor stimulation, regulates murine macrophage inflammatory responses. Proceedings of the National Academy of Sciences of the United States of America 2009, 106, $15819-15824$.

[41] Turroni, F., Taverniti, V., Ruas-Madiedo, P., Duranti, S., et al., Bifidobacterium bifidum PRL2010 modulates the host innate immune response. Applied and environmental microbiology 2014, 80, 730-740.

[42] Hoffmann, M., Rath, E., Holzlwimmer, G., Quintanilla-Martinez, L., et al., Lactobacillus reuteri 100-23 transiently activates intestinal epithelial cells of mice that have a complex microbiota during early stages of colonization. The Journal of nutrition 2008, 138, $1684-1691$

[43] Dykstra, N. S., Hyde, L., Adawi, D., Kulik, D., et al., Pulse probiotic administration induces repeated small intestinal Muc3 expression in rats. Pediatric research 2011, 69, 206211.

[44] Zhou, Y. D., Barnard, M., Tian, H., Li, X., et al., Molecular characterization of two mammalian bHLH-PAS domain proteins selectively expressed in the central nervous system.

This article is protected by copyright. All rights reserved. 
Proceedings of the National Academy of Sciences of the United States of America 1997, 94, 713-718.

[45] Zhao, J., Du, F., Shen, G., Zheng, F., Xu, B., The role of hypoxia-inducible factor-2 in digestive system cancers. Cell Death Dis 2015, 6, e1600.

[46] Giatromanolaki, A., Sivridis, E., Maltezos, E., Papazoglou, D., et al., Hypoxia inducible factor 1 alpha and 2 alpha overexpression in inflammatory bowel disease. J Clin Pathol 2003, $56,209-213$.

[47] Xue, X., Ramakrishnan, S., Anderson, E., Taylor, M., et al., Endothelial PAS Domain Protein 1 activates the inflammatory response in the intestinal epithelium to promote colitis in Mice. Gastroenterology 2013, 145, 831-841.

[48] Mastrogiannaki, M., Matak, P., Keith, B., Simon, M. C., et al., HIF-2 alpha, but not HIF1 alpha, promotes iron absorption in mice. J Clin Invest 2009, 119, 1159-1166.

[49] Das, N., Xie, L., Ramakrishnan, S. K., Campbell, A., et al., Intestine-specific disruption of Hypoxia-Inducible Factor (HIF)-2 alpha improves anemia in sickle cell disease. The Journal of biological chemistry 2015, 290, 23523-23527.

[50] Davis, M. E., Heidel, J. D., Liu, J. Y. C., U.S. Patent No. 8,114,983. 2012.

[51] Scheuermann, T. H., Li, Q., Ma, H. W., Key, J., et al., Allosteric inhibition of hypoxia inducible factor-2 with small molecules. Nat Chem Biol 2013, 9, 271-276.

This article is protected by copyright. All rights reserved. 


\section{Figure legends}

Figure 1. Strain- and time-specific expression of miR-148a in Caco-2 intestinal cells in response to bifidobacteria.

Relative expression in intestinal Caco-2 cells incubated in presence of Bifidobacterium strains for 1, 4 and 24 hours. Data are presented as relative fold change in Caco- 2 cells coincubated with one of the three strains (B. bifidum MIMBb75, B. bifidum NCC390 or $B$ longum NCC2705) compared to sterile vehicle with delta Cts normalized to RNU6B. Significance is based on ANOVA plus Tukey's multiple comparisons test. ${ }^{*} \mathrm{P}<.05, * * \mathrm{P}<.01$ and $* * * \mathrm{P}<.001$. The average of three biological replicates with corresponding standard deviations are presented.

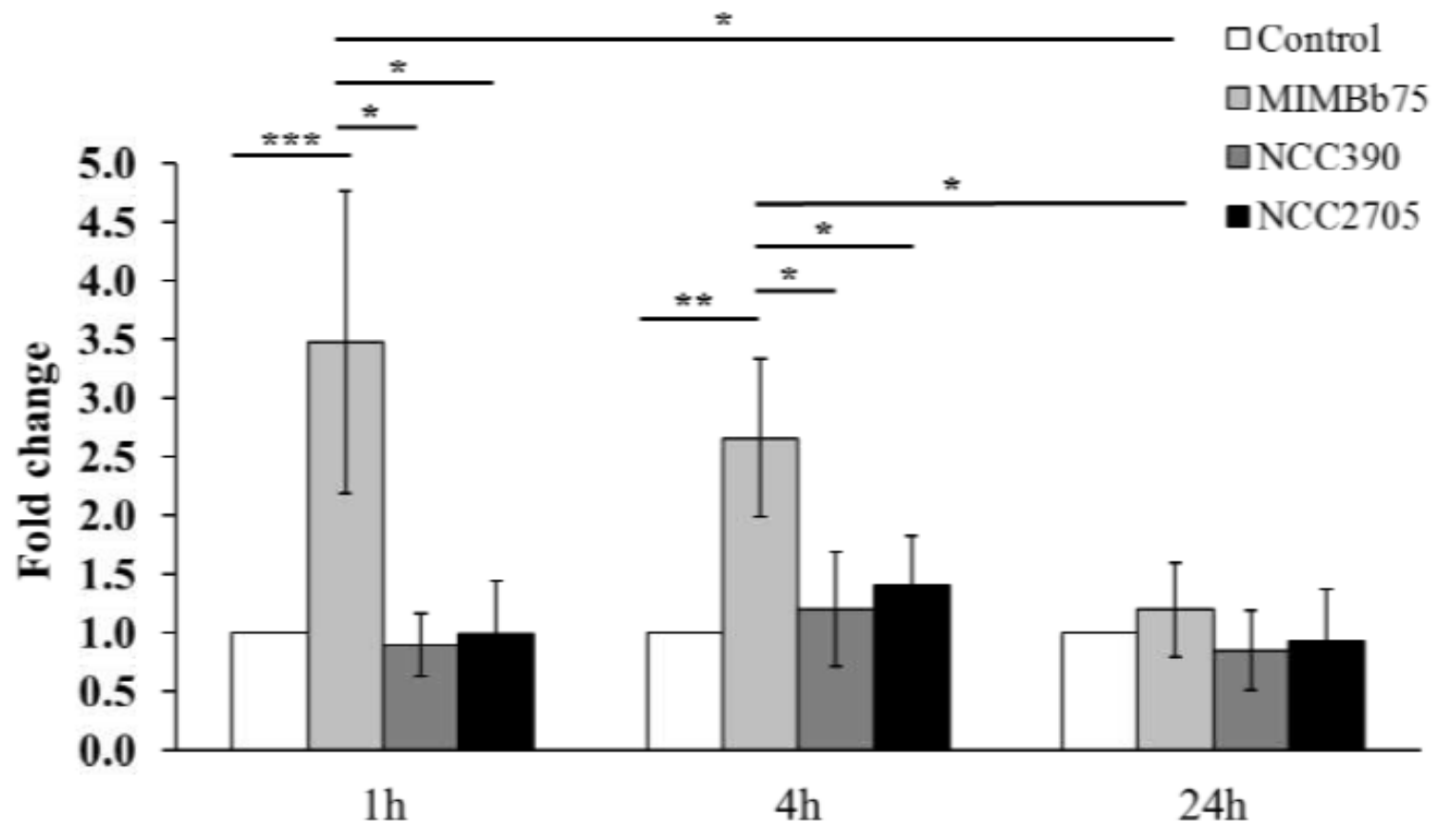

This article is protected by copyright. All rights reserved. 
Figure 2. The expression of miR-148a increases early in mouse cecum following administration of $B$. bifidum MIMBb75

Relative expression in mice cecum following intragastric gavage of either B. bifidum MIMBb75 or sterile PBS for two and fourteen days in study 1 (A) and or for two days in study 2 (B). Data are presented as relative fold change in B. bifidum MIMBb75 versus control group, with delta Cts normalized to snoRNA-135. Significance is based on non-parametric Mann-Whitney test. $* \mathrm{P}<.05, * * \mathrm{P}<.01$ and $* * * \mathrm{P}<.001$. The average of 4 to 7 mice per group corresponding standard deviations are presented.
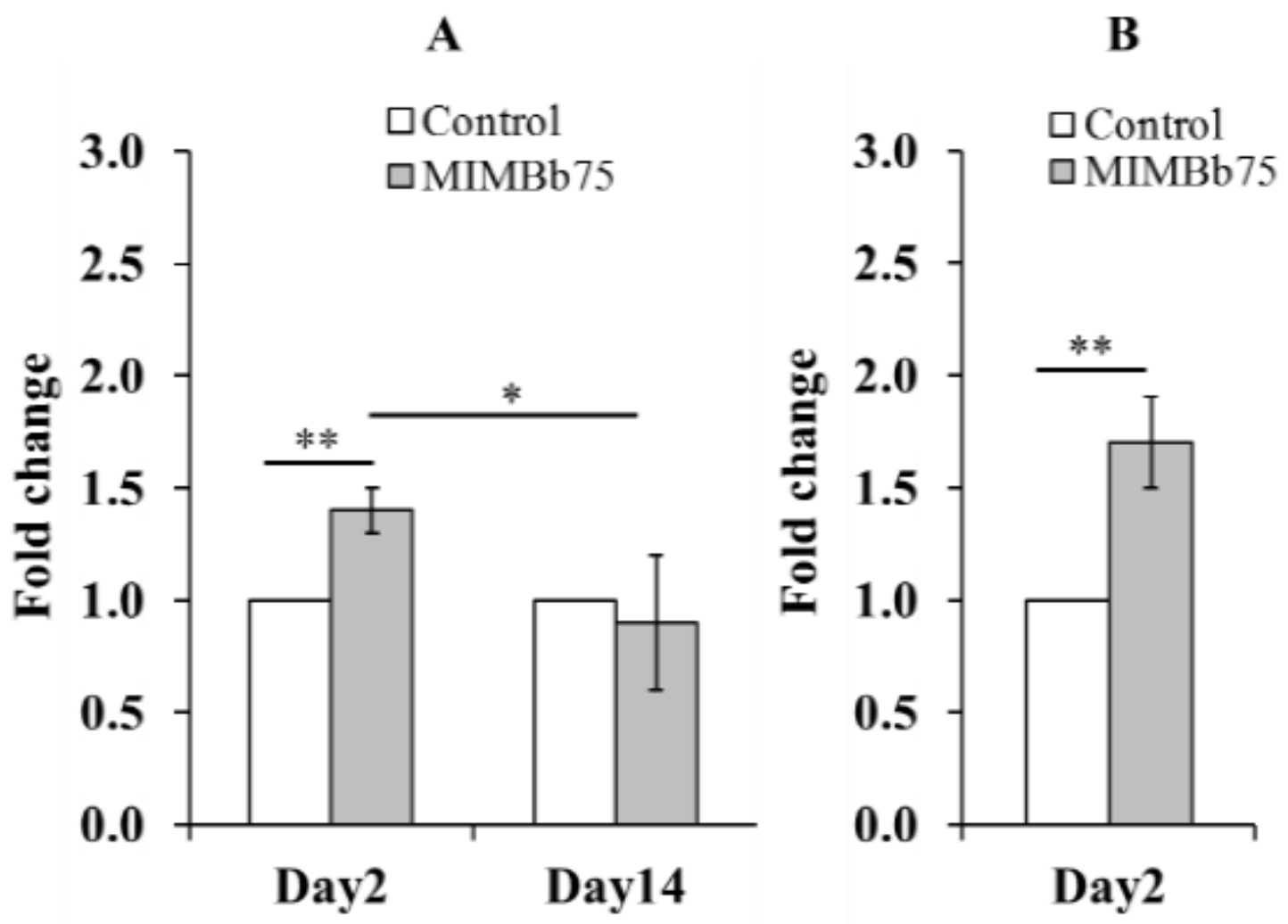

This article is protected by copyright. All rights reserved. 
Figure 3. B. bifidum MIMBb75 modifies the expression of silenced miR-148a in Caco-2 cells

Relative expression of miR-148a in transfected Caco-2 cells co-incubated B. bifidum MIMBb75 or sterile vehicle for 1, 4 and 24 hours. Data are presented as relative fold change in a) miR-148a inhibitor transfected Caco-2 cells incubated with vehicle only (miR-148a inhibitor), b) miR-148a inhibitor transfected Caco-2 cells incubated with $B$. bifidum MIMBb75 (miR-148a inh+MIMBb75) and c) mock transfected Caco-2 cells incubated with B. bifidum MIMBb75 (MIMBb75 only), with delta Cts normalized to RNU6B. Significance is based on ANOVA plus Tukey's multiple comparisons test. ${ }^{*} \mathrm{P}<.05,{ }^{*} \mathrm{P}<.01$ and *** $\mathrm{P}<.001$. The average of three biological replicates with corresponding standard deviations are presented.

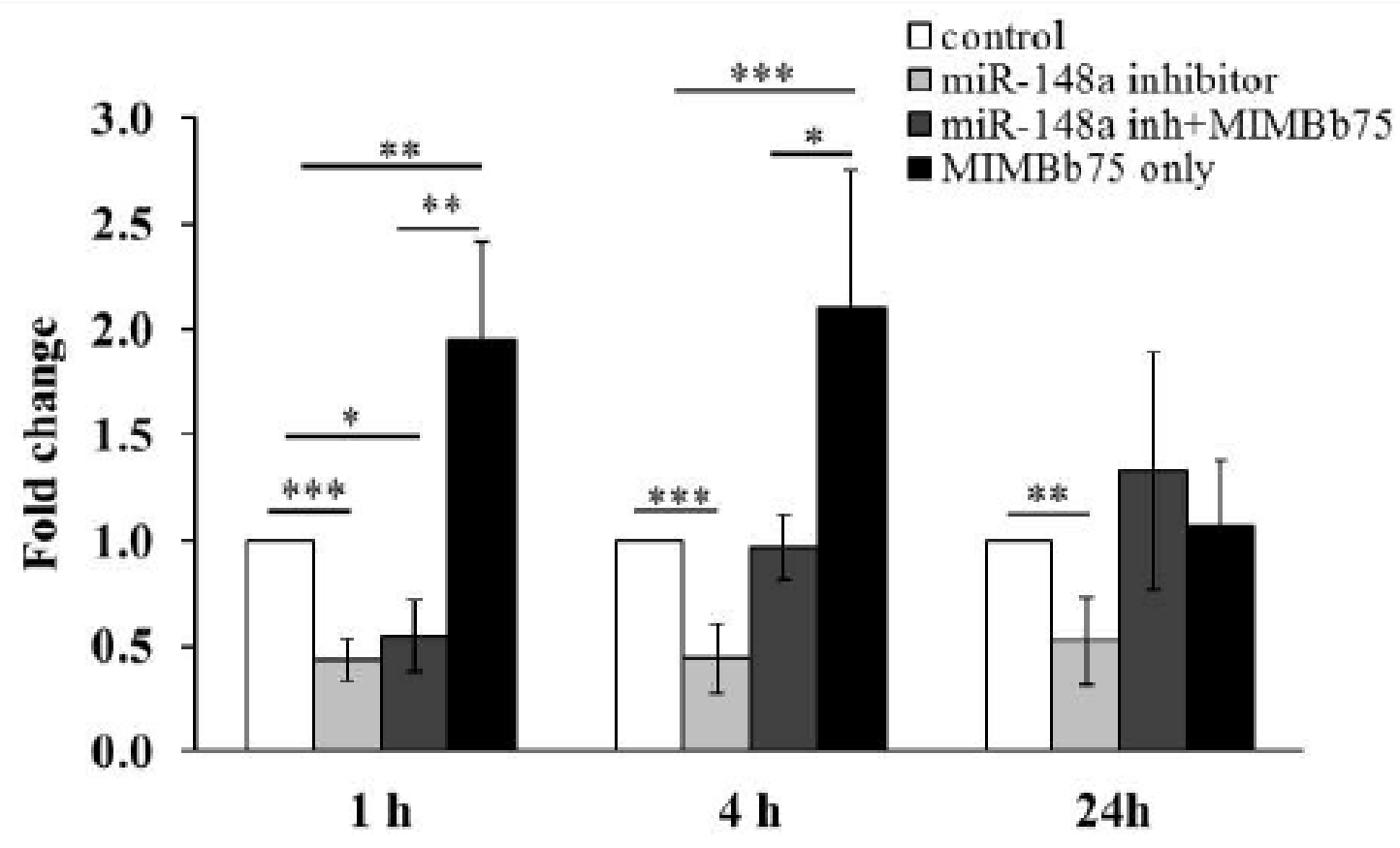

This article is protected by copyright. All rights reserved. 
Figure 4. B. bifidum MIMBb75 downregulates EPAS1 in intestinal cells via miR-148a in a time-dependent manner.

Relative expression of EPAS1 in transfected Caco-2 cells co-incubated with B. bifidum MIMBb75 or sterile vehicle for 1, 4 and 24 hours (A). Data are presented as relative fold change in a) miR-148a inhibitor transfected Caco-2 cells incubated with vehicle only (miR148a inhibitor), b) miR-148a inhibitor transfected Caco-2 cells incubated with B. bifidum MIMBb75 (miR-148a inh+MIMBb75) and c) mock transfected Caco-2 cells incubated with B. bifidum MIMBb75 (MIMBb75 only), with delta Cts normalized to RNU6B. Significance is based on ANOVA plus Tukey's multiple comparisons test. $* \mathrm{P}<.05$, ** $\mathrm{P}<.01$ and *** $\mathrm{P}<.001$. The average of three biological replicates with corresponding standard deviations are presented. Relative expression in mice cecum receiving either B. bifidum MIMBb75 or sterile PBS by intragastric gavage for two days (B). Data are presented as relative fold change in B. bifidum MIMBb75 versus control group, with delta Cts normalized to Actb. Significance is based on non-parametric Mann-Whitney test. ${ }^{*} \mathrm{P}<.05$. The average of 4 to 6 mice per group corresponding standard deviations are presented.
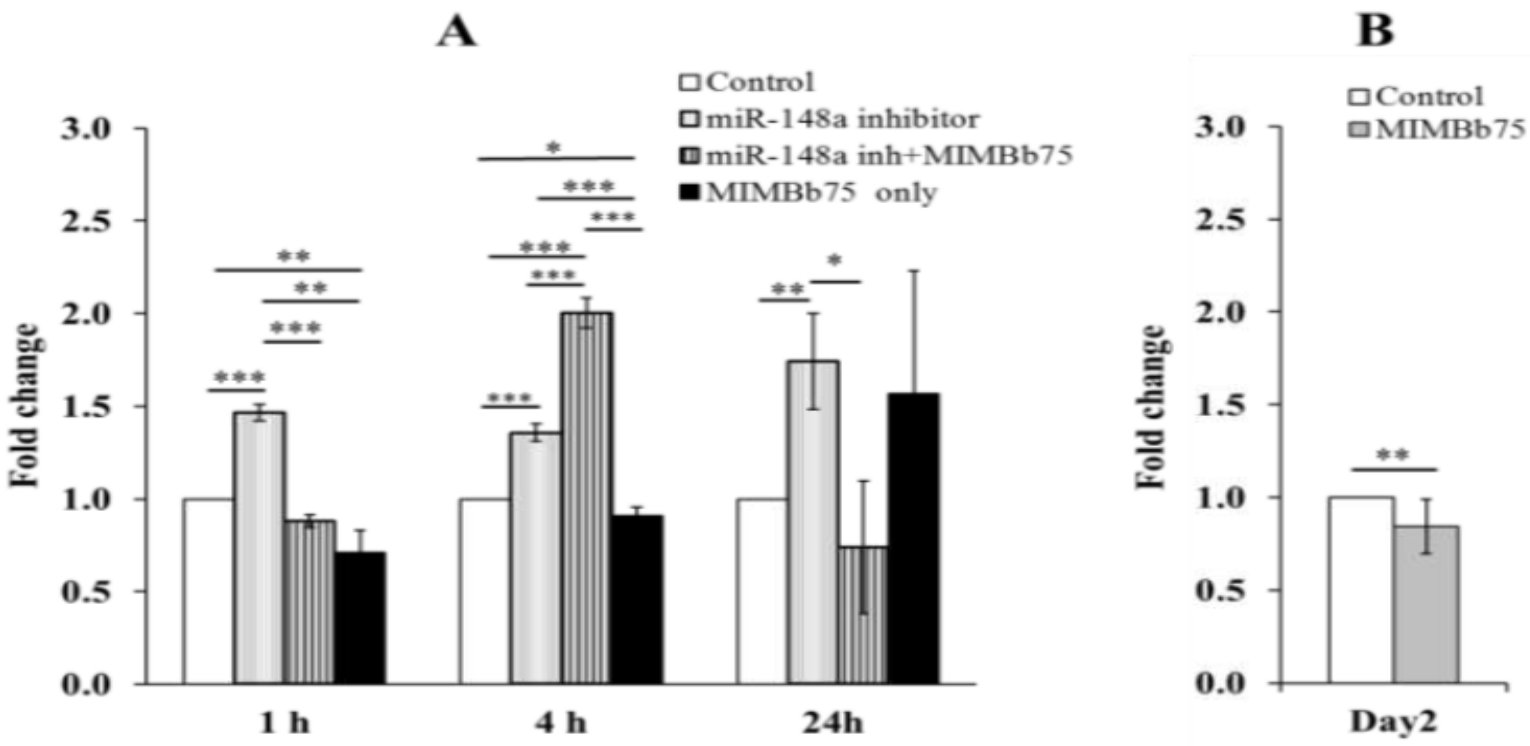

This article is protected by copyright. All rights reserved. 


\section{Graphical abstract}

We studied if probiotic bifidobacteria alter intestinal microRNA and downstream target gene response. We found that intestinal cells respond to Bifidobacterium bifidum exposure via increased expression of miR-148a in a time-dependent manner. At 1 hour, increase of miR148a results in decreased expression of its target gene endothelial PAS domain protein 1. EPAS1 is involved in intestinal cancers and inflammation; thus, B. bifidum could provide a strategy to support homeostasis via microRNA.

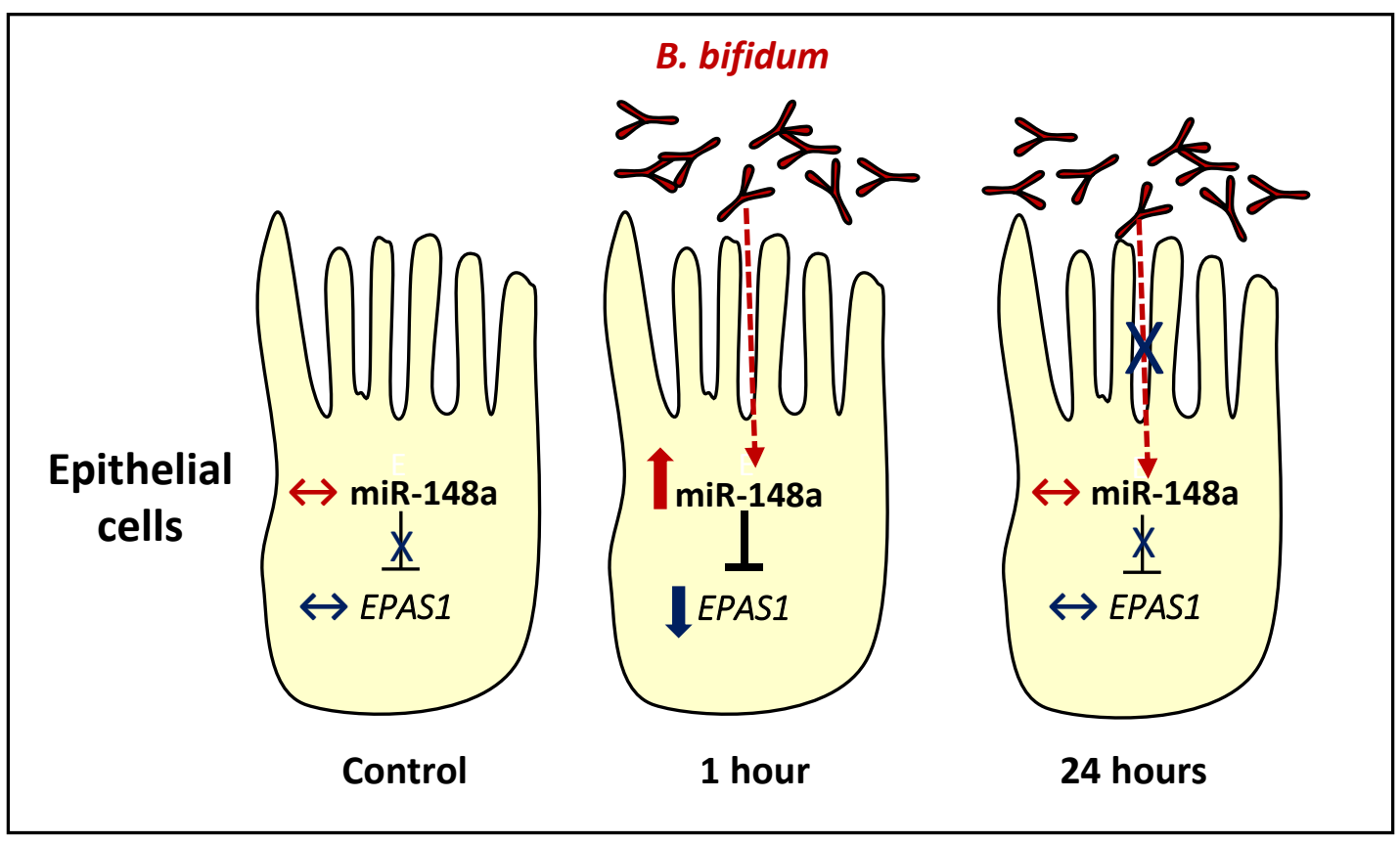

This article is protected by copyright. All rights reserved. 